\title{
Perfil clínico-funcional dos idosos de uma instituição de longa permanência
}

\author{
Clinical-functional profile of the elderly in an institution of long stay \\ Perfil clínico-funcional del mayor en una institución de larga estancia
}

Gisele da Silva Cardoso Fernandes ${ }^{1 *}$, Maria de Fátima Cândida da Silva ${ }^{1}$, Adriane Bonotto Salin ${ }^{1}$, Isabele Cristina Mascarenhas Costa ${ }^{1}$, Thaís Millena Cardoso de Lima ${ }^{1}$, Gabriela Aparecida Paz de Castro Barreto ${ }^{1}$, Thainá França Oliveira ${ }^{1}$, Maria Michelle Pereira dos Santos ${ }^{1}$.

\begin{abstract}
RESUMO
Objetivo: Avaliar o perfil clínico-funcional dos idosos de uma instituição de longa permanência na região Norte do Brasil. Métodos: Tratou-se de uma pesquisa de campo com abordagem quantitativa e exploratória, obedecendo aos aspectos éticos e legais, determinado pela Resolução 466/12 do Conselho Nacional de Saúde. Foi utilizado um questionário para descrição do perfil sociodemográfico e clínico. As informações colhidas foram analisadas e tabuladas por intermédio de uma planilha eletrônica no programa Epi-info, versão 7.2.2. Resultados: A maioria dos idosos apresentaram baixa escolaridade e idade entre 70 a 79 anos (54,55\%). A maioria, do sexo masculino $(81,82 \%$,) , solteiro $(86,36 \%)$ e sem filhos $(72,73 \%)$; e $(59,09 \%)$ tem mais de 5 anos que residem na instituição e $54,55 \%$ não possuem contato com o familiar; $95,45 \%$ dos idosos apresentam algum tipo de doença crônica, 90,91\% três ou mais tipo de comorbidades e 40,91\% utilizam mais de sete medicamentos diferentes. Conclusão: $O$ estudo possibilitou o conhecimento do perfil clínico-funcional de alguns idosos residentes da Instituição de Longa Permanecia e irá contribuir para o planejamento de melhorias na qualidade de vida do público avaliado.
\end{abstract}

Palavras-chave: Idosos, Mobilidade funcional, Instituição de longa permanência.

\begin{abstract}
Objective: To evaluate the clinical and functional profile of elderly people in a long-term institution in the North of Brazil. Methods: This was a field research with a quantitative and exploratory approach, complying with ethical and legal aspects, determined by Resolution 466/12 of the National Health Council. A questionnaire was used to describe the sociodemographic and clinical profile. The information collected was analyzed and tabulated using an electronic spreadsheet in the Epi-info program, version 7.2.2. Results: Most seniors had low education and aged between 70 and 79 years $(54.55 \%)$. The majority were male $(81.82 \%)$, single $(86.36 \%)$ and without children $(72.73 \%)$; and $(59.09 \%)$ have lived in the institution for more than 5 years and $54.55 \%$ have no contact with the family member; $95.45 \%$ of the elderly have some type of chronic disease, $90.91 \%$ have three or more types of comorbidities, and $40.91 \%$ use more than seven different medications. Conclusion: The study enabled the knowledge of the clinical and functional profile of some elderly residents of the Long Stay Institution and will contribute to the planning of improvements in the quality of life of the assessed public.
\end{abstract}

Keywords: Elderly, Functional mobility, Long-term institution.

\section{RESUMEN}

Objetivo: Evaluar el perfil clínico y funcional de ancianos en una institución de larga duración en el norte de Brasil. Métodos: Se trató de una investigación de campo con abordaje cuantitativo y exploratorio, cumpliendo con los aspectos éticos y legales determinados por la Resolución 466/12 del Consejo Nacional de Salud, se utilizó un cuestionario para describir el perfil sociodemográfico y clínico. La información recolectada fue analizada y tabulada mediante una hoja de cálculo electrónica en el programa Epi-info, versión 7.2.2. Resultados: La mayoría de los adultos mayores tenían un nivel educativo bajo y tenían entre 70 y 79 años (54,55\%). La mayoría eran hombres $(81,82 \%)$, solteros $(86,36 \%)$ y sin hijos $(72,73 \%)$; y $(59.09 \%)$ ha vivido en la institución por más de 5 años y $54.55 \%$ no tiene contacto con el familiar; El 95,45\% de los ancianos tiene algún tipo de enfermedad crónica, el 90,91\% tiene tres o más tipos de comorbilidades y el 40,91\% usa más de siete medicamentos diferentes. Conclusión: El estudio permitió conocer el perfil clínico y funcional de algunos ancianos residentes de la Institución de Larga Estancia y contribuirá a la planificación de mejoras en la calidad de vida del público evaluado.

Palabras clave: Anciano, Movilidad funcional, Institución de larga duración.

${ }^{1}$ Faculdade Interamericana de Porto Velho - UNIRON, Porto Velho - RO. *E-mail: gysellycardoso@hotmail.com

SUBMETIDO EM: 6/2021

ACEITO EM: 6/2021

PUBLICADO EM: 6/2021 


\section{INTRODUÇÃO}

O envelhecimento é um processo natural que ocorre ao longo de nossas vidas de maneira progressiva e irreversível, e pode ser definido como um conjunto de alterações morfológicas, fisiológicas e bioquímicas, que podem sofrer a influência de fatores sociais, políticos, econômicos e psicológicos (SANTOS AO, et al., 2017).

A senilidade é descrita como uma experiência heterogênea, que dependerá de como o indivíduo organiza sua vida com base em circunstâncias histórico-culturais, incidência de patologias e interação entre genética e problemas ambientais. Embora haja grandes diferenças individuais nesse processo, três domínios gerais devem ser considerados: aumento do déficit físico; pressões e perdas sociais; e perspectiva iminente de finitude. Assim, mesmo que o envelhecimento bem-sucedido seja multifacetado, a maioria dos idosos experimentará os domínios acima (BELEZA CMF e SOARES SM, 2019).

O envelhecimento é uma realidade mundial e é considerado como um dos grandes desafios da saúde pública, pois o aumento da morbimortalidade decorrente das doenças crônicas degenerativas que comprometem a qualidade de vida do idosos. Outro ponto relevante sobre o envelhecimento é o abandono pelos familiares nas Instituição de Longa Permanência (ILP), seja pelo fato de não possuírem o suporte necessário para cuidar de uma pessoa com idade avançada em casa (ALARCON MSF, et al., 2019).

No Brasil as ILPI no decorrer da história aparecem ligadas a um serviço onde pessoas idosas pobres, sem famílias e doentes eram abrigadas e dirigidas por irmãs de caridade. No entanto a Resolução da Diretoria Colegiada (RDC) no 283/2005, define ILPI como instituições de caráter residencial destinada a domicílio coletivo de indivíduos com idade igual ou superior a 60 anos, com ou sem suporte familiar (ROSA VPP, et al., 2019). Para atender esta população, a equipe da ILPI necessita realizar uma avaliação multivariada, a fim de conhecer aspectos biopsicossociais, valores, crenças, sentimentos e necessidades de cuidados, além de fatores sociodemográfico, funcionais e cognitivos, a fim de implementar assistência de cada idoso de maneira holística e levando ao bem-estar e satisfação dos idosos (RIBEIRO EG, et al., 2018).

A elaboração do perfil clínico-funcional é baseado nas atividades de vida diária (AVD), que são divididas em: atividade básica de vida diária (ABVD), relacionada à atenção de conservação (alimentar-se, vestir-se, tomar banho, deambular, controlar esfíncteres); as atividades instrumentais de vida diária (AIVD), que impedem deveres que autorizam a conservação da vida dos institucionalizados (realizar compras, utilizar telefone, geri assuntos econômicos etc.) e as atividades avançadas de vida diária (AAVD), mas incompreensível, que separam dominação corporal, lazer, social e produtivo (ANTUNES C, et al., 2017).

Assim, de acordo com Santos AO, et al. (2017) em um ambiente institucional, é necessário que os idosos adquiram novas experiências, com diferentes hábitos, regras, horários e relações sociais. As mudanças de institucionalização geralmente podem levar a mudanças de comportamento e identidade, levando-as a viver em um mundo privado, com uma perda de individualidade e uma tendência ao isolamento.

Neste sentido, surge a seguinte questão da pesquisa: Qual é o perfil clínico-funcional dos idosos institucionalizados no Norte do Brasil? Para responder a esse questionamento, delineou-se o seguinte objetivo: Avaliar o perfil clínico-funcional dos idosos residentes em uma Instituição de Longa Permanência localizada em um município da região Norte do Brasil.

\section{MÉTODOS}

Trata-se de uma pesquisa de campo com abordagem quantitativa e exploratória, obedecendo aos aspectos éticos e legais do estudo, determinado pela Resolução 466/12, do Conselho Nacional de Saúde. Foi utilizado um questionário para descrição do perfil sociodemográfico e clínico bem como a utilização de um instrumento validado denominado Escala de Katz (avaliar os aspectos funcionais relacionados às Atividades de Vida Diária e para o levantamento de grau de dependência dos idosos) para a coleta de dados. As informações colhidas foram analisadas e tabuladas por intermédio de uma planilha eletrônica, no programa Epi-info, versão 7.2.2. A pesquisa foi aprovada pelo Comitê de Ética de Pesquisa, com o parecer de n.ำ 4.251.093. 
A população do estudo foi constituída por idosos de ambos os sexos, residentes na ILPI. A definição da amostra foi realizada de forma não probabilística, por acessibilidade no qual foram convidados a participar da pesquisa todos os idosos residentes na ILPI. De acordo com a Lei no 8.842 no Art. 2 - Considera-se idoso, para os efeitos desta lei, a pessoa maior de sessenta anos de idade.

Participaram da coleta, idosos que aceitaram participar do estudo, sendo homens e mulheres e foram excluídos os idosos que não estavam em condições física e mentais para a aplicação dos instrumentos e os que não aceitaram seguir os comandos da aplicação do instrumento.

\section{RESULTADOS}

Participaram deste estudo 22 idosos de ambos os sexos e idade, residentes em uma ILP. A coleta de dados foi realizada no mês de dezembro de 2020 e foi constatada a predominância de pacientes do sexo masculino $81,82 \%$ com idade de 70 a 79 anos $54,55 \%$ e $59,09 \%$ fazem mais de 5 anos que residem na ILPI. Além disso, observou-se que $86,36 \%$ são solteiros, $45,45 \%$ não são alfabetizados, $77,27 \%$ recebem benefício assistencial, $72,73 \%$ não possuem filhos, $54,55 \%$ não possuem contato com o familiar (Tabela 1).

Tabela 1 - Perfil Sociodemográfico dos idosos de uma Instituição de Longa Permanência.

\begin{tabular}{|c|c|c|}
\hline Variáveis & $\mathbf{N}$ & $\%$ \\
\hline \multicolumn{3}{|l|}{ Idade } \\
\hline 60 a 69 anos & 4 & 18,18 \\
\hline 70 a 79 anos & 12 & 54,55 \\
\hline 80 anos ou mais & 16 & 27,27 \\
\hline \multicolumn{3}{|l|}{ Sexo } \\
\hline Feminino & 4 & 18,18 \\
\hline Masculino & 18 & 81,82 \\
\hline \multicolumn{3}{|l|}{ Nacionalidade } \\
\hline Brasileiro & 21 & 95,45 \\
\hline Italiano & 1 & 4,55 \\
\hline \multicolumn{3}{|l|}{ Estado Civil } \\
\hline Divorciado & 2 & 9,09 \\
\hline Solteiro & 19 & 86,36 \\
\hline Viúvo & 1 & 4,55 \\
\hline \multicolumn{3}{|l|}{ Escolaridade } \\
\hline Não Alfabetizado & 10 & 45,45 \\
\hline Alfabetizado & 6 & 27,27 \\
\hline Fundamental incompleto & 5 & 22,73 \\
\hline Fundamental completo & 1 & 4,55 \\
\hline \multicolumn{3}{|l|}{ Situação Previdenciária } \\
\hline Não tem & 1 & 4,55 \\
\hline Aposentado & 1 & 4,55 \\
\hline BPC (Benefício de Prestação Continuada) & 17 & 77,27 \\
\hline BPC (em análise) & 3 & 13,64 \\
\hline \multicolumn{3}{|l|}{ Possui contato familiar } \\
\hline Não & 12 & 54,55 \\
\hline Sim & 10 & 45,45 \\
\hline \multicolumn{3}{|l|}{ Possui Filhos } \\
\hline Não & 16 & 72,73 \\
\hline 1 á 2 & 4 & 18,18 \\
\hline 4 filhos ou mais & 2 & 9,09 \\
\hline \multicolumn{3}{|l|}{ Tempo de Instituição } \\
\hline Menos de 1 ano & 4 & 18,18 \\
\hline De 1 a 3 anos & 5 & 22,73 \\
\hline 5 anos ou mais & 13 & 59,09 \\
\hline
\end{tabular}

Fonte: Fernandes GCS, et al., 2021. 
Em relação ao perfil clínico dos idosos institucionalizados, a tabela mostra que $95,45 \%$ dos idosos apresentam algum tipo de doença crônica 40,91\% utilizam mais de 7 tipos de medicamentos. Em relação a quedas sofridas nos últimos seis meses a tabela nos mostra que $95,45 \%$ não obtiveram quedas. Apenas $36,36 \%$ dos idosos não fazem uso de nenhum tipo de dispositivo de marcha; os demais $63,64 \%$ utilizam cadeiras de rodas, bengalas muletas ou são acamados. Em relação as comorbidades os idosos apresentam em maioria $90,91 \%$ três ou mais tipo de comorbidades (Tabela 2).

Tabela 2 - Perfil clínico dos idosos de uma Instituição de Longa Permanência.

\begin{tabular}{lcc}
\hline Variáveis & N & $\%$ \\
\hline Possui doenças crônicas & 1 & \\
\hline Não & 21 & 4,55 \\
Sim & & 95,45 \\
\hline Uso de Medicamentos & 2 & \\
\hline 1 a 2 & 8 & 9,09 \\
3 a 4 & 3 & 36,36 \\
5 a 6 & 9 & 13,64 \\
7 ou mais & & 40,91 \\
\hline Queda nos últimos 6 meses & 21 & \\
\hline Não & 1 & 95,45 \\
Sim & & 4,55 \\
\hline Uso de dispositivo de marcha & 8 & \\
\hline Não & 1 & 36,36 \\
Acamado & 2 & 4,55 \\
Andador & 2 & 9,09 \\
Bengala & 8 & 9,09 \\
Cadeira de roda & 1 & 36,36 \\
Muleta & & \\
\hline Comorbidades & 1 & 4,55 \\
\hline Uma & 1 & 4,55 \\
Duas & 20 & 4,55 \\
Três ou mais & & 90,91 \\
\hline
\end{tabular}

Fonte: Fernandes GCS, et al., 2021.

O instrumento realizado com os idosos denominado escala de Katz nos mostra que $53,33 \%$ dos idosos são totalmente independentes em idades superiores a 75 anos, $46,67 \%$ são de dependência parcial, na faixa etária de 60 a 75 anos. Nesta tabela nos mostra que não há associação entre as variáveis, grau de independência (escala de Katz), doenças crônicas e idade (Tabela 3).

Tabela 3 - Análise entre as variáveis de idades, doenças crônicas e grau de dependência (Escala de Katz) em idosos institucionalizados.

\begin{tabular}{|c|c|c|c|c|c|}
\hline \multirow{3}{*}{ Variáveis } & $\mathbf{N}$ & $\%$ & $\mathbf{N}$ & $\%$ & \multirow{3}{*}{ Valor $-p$} \\
\hline & \multicolumn{4}{|c|}{ Idade } & \\
\hline & \multicolumn{2}{|c|}{60 a 75 anos } & \multicolumn{2}{|c|}{ Acima de 75 anos } & \\
\hline \multicolumn{6}{|l|}{ Escala de Katz } \\
\hline Dependência & 4 & 54,14 & 3 & 42,86 & \multirow{2}{*}{1,000} \\
\hline Total Dependência & 7 & 46,67 & 8 & 53,33 & \\
\hline \multicolumn{6}{|l|}{ Doenças Crônicas } \\
\hline Sim & 0 & 0,00 & 1 & 100 & \multirow{2}{*}{1,000} \\
\hline Não & 11 & 52,38 & 10 & 47,62 & \\
\hline
\end{tabular}

Fonte: Fernandes GCS, et al., 2021. 


\section{DISCUSSÃO}

No estudo verificou-se que dos 22 idosos avaliados, a distribuição em relação ao sexo teve predomínio de idosos do sexo masculino $81,82 \%$, dado divergente encontrado por Rosa VPP, et al. (2019) em estudo desenvolvido em ILPI's de Porto Alegre - RS, onde a maioria dos participantes eram do sexo feminino. No entanto ao avaliar o tempo de tempo de permanência e a escolaridade dos participantes do estudo no RS, os resultados corroboram com achados nessa pesquisa, 59,09\% apresentam mais de 5 anos de institucionalizados e $45,45 \%$ não são alfabetizados, dados semelhantes ao estudo desenvolvido por Rosa VVP, et al. (2019) onde um número significativo de idosos analfabetos e mais da metade dos residentes tem mais de 5 anos na instituição.

Acredita-se que a diferença em relação no sexo possa estar associada ao fato dos idosos serem solteiros, divorciados, ou seja a ausência de um companheiro e o apoio social e familiar enfraquecido, uma vez que Fluetti MT, et al. (2018) apontam estes fatores com fatores que possam ser responsáveis pela institucionalização do idoso.

Quanto a faixa etária a maioria dos idosos apresentou idade entre 70 a 79 anos (54,55\%),dados estes divergentes dos estudos de Souza FJM, et al. (2020) e Fluetti MT, et al. (2018) o primeiro estudo, realizado com grupo de idosos de uma ILP, apontou idosos com uma idade superior à da pesquisa, a média foi de $69,3 \%$, entre a faixa etária de mais de 80 anos, o segundo estudo confirma dados levantados pelo estudo anterior, realizado em Juiz de Fora, MG, verificou-se que $55,7 \%$ dos idosos institucionalizados possuíam 80 anos ou mais, caracterizando os idosos da pesquisa uma população de idosa jovem.

No perfil clínico levantado, destaca - se o quantitativo do uso de medicamentos com (40,91\%) utilizam sete ou mais medicamentos os quais são denominados como polifarmácia; e $(36,365)$ utilizam de três a quatro tipos de medicações. Em estudo levantado por Nascimento RCRM, et al. (2017) o autor destaca que em estudo realizado com idosos na faixa etária acima de 65 anos é de $32,9 \%$ a prevalência de polifarmácia.

A respeito da variável escolaridade a maioria,68,18\%, dos pesquisados são analfabetos, ou não concluíram o ensino fundamental, dados este que corroboram com estudo realizado na ILP do Lar São Francisco de Assis em Simão Dias - SE por Santana JDM, et al. (2018) onde descrevem que $70 \%$ dos idosos são analfabetos ou não possuem o ensino fundamental incompletos.

A prevalência da baixa escolaridade pode estar relacionada primordialmente, a condução de situações de seu ambiente social, a não convivência com pessoas sem acesso ao ambiente escolar durante a infância, ou seja, a baixa escolaridade comum na população idosa, principalmente na institucionalizada tem relação a com o que estes idosos vivenciaram na infância, um estágio social em que o ensino não era tido como uma prioridade. Flores JR, et.al (2021) em estudo desenvolvidos em duas ILPI, no município de Santa Maria/RS, além de apresentar dados que vem confirmar nossos achados neste estudo, pois mais de $70 \%$ dos internos das instituições não possuem ensino fundamental completou ou são analfabetos, e este fator pode estar relacionado às dificuldades de acesso ao ambiente escolar do passado correlacionadas às atividades agrícolas.

Alcântara RKL, et al. (2019) descrevem que a baixa escolaridade é um achado muito comum e frequente ao avaliar ILPI, uma vez em que decorre da realidade passada de discriminação de oportunidades educacionais para com esses idosos. No Brasil reflete-se, a associação entre as condições socioeconômicas, com a conclusão da educação primária e secundária e acesso a formação universitária.

Em relação ao vinculo familiar $86,36 \%$ dos idosos são solteiros e $72,73 \%$ não possuem filhos, e quanto ao contato e manutenção do vínculo familiar $54,55 \%$ dos pesquisados não possuem nenhum contato.

Leite AK, et al. (2020) em estudo realizado com idosos institucionalizados de Araçatuba - SP- onde descreve que idoso tem uma imagem de "depósito de pessoas idosas" das institucionalizações após serem levados muitas vezes por próprio membro familiar ou de qualquer natureza, na qual acaba por desperta sentimento de desprezo e abandono, e isso acaba dificultando um vínculo familiar.

Haddad PCM e Calamita Z (2020) descrevem que o aumento de idosos na população, agregado a uma crescente dependência, mudanças socioeconômicas e culturais na sociedade moderna, à baixa renda, além 
das alterações na constituição familiar são alguns fatores predisponentes ao processo de institucionalização do idoso, e afirma que a ausência de filhos, de um companheiro, comprometimento cognitivo e dependência para as atividades de vida diária predispõe o idoso a institucionalização conforme dados encontrado neste estudo.

O fato de o vínculo familiar estar comprometido nos internos da ILPI, onde mais da metade dos internos não tem contato com familiares pode estra relacionado a situações que tenham transcorrido com o idoso antes do envelhecimento. Rohde J e Coutinho AS (2020) descrevem a internação do familiar possa estar relacionado a questões financeiras, ou quando os familiares necessitam trabalhar e não conseguem cuidar do idoso, assim como também por problemas de vínculos familiares, ou seja, quando o vínculo da família para com o ancião já apresenta fragilidades por determinado motivo que tenham acontecido antes do envelhecimento.

Cataneo FM, et al. (2019) apontam que embora a família ainda seja uma alternativa para o apoio ao idoso, os autores relatam que existe incontáveis razões para interferir na persistência dos idosos com suas famílias, como conflito familiar, emergência de problemas de saúde, dependência física do idoso, membro familiar que está ocupado com o trabalho diário, sem tempo para fornece cuidados e companhias, além de não ter como ofertar um espaço de convivência e socialização.

Ao investigar sobre presença de comorbidades, 90, 91\% idosos pesquisados apresentam três ou mais comorbidades e dentre elas $95,45 \%$ são portadores algum tipo de doença crônica.

Segundo China DL, et al (2021) a população idosa está envelhecendo de uma forma crescente, e o envelhecimento faz analogia direta com a incidência das patologias crônicas não transmissíveis, e por vezes a pouca qualidade de vida pode potencializar a vulnerabilidade a doenças crônicas e degenerativas. Para Scortegagna HM, et al. (2021) à transição epidemiológica que está ocorrendo, somada a heterogeneidade do segmento idoso configura um desafio devido representar expressiva probabilidade da ocorrência de doenças crônicas não transmissíveis, vulnerabilidade e outras fragilidades, que acometem especialmente o segmento de maior idade da população

Nos idosos em estudo houve uma predominância de Doenças crônicas não transmissíveis (DCNT) em idosos do sexo masculino com 100,00\% na faixa etária acima de 75 anos, enquanto a do sexo feminino apresenta $75,00 \%$ na faixa etária de 60 a 75 anos. Figueiredo AEB, et al. (2021) ressalta que DCNT tem uma série de características patológicas as razões e os fatores de risco são muitos, o período de incubação é longo e o curso da doença é prolongado. Além disso são de origem não infecciosa e podem causar disfunções.

Identificando-se com isso vem tendo o motivo real que os idosos têm um grande alto índice de doenças crônicas por terem as piores condições socioeconômicas que estão associadas pela perda da capacidade física e funcional entre esses idosos, pois assim eles acabam acumulando uma maior carga de doenças ao longo da vida, desempenhando atividades laborais insalubres, possuindo hábitos de vida prejudiciais e menor acesso aos serviços de saúde.

O índice de doenças crônicas em idosos do sexo masculina, uma vez que a maior parte dos homens não se prepara para alcançar a o envelhecimento, são as mulheres que são vistas como cuidadoras de si mesmo, tem mais paciência, vai rotineiramente ao atendimento médico e desfrute de maior disponibilidade o que pode estar relacionado à chamada vulnerabilidade feminizarão, mas também por estímulo, porque ainda jovem, são incentivadas a irem ao médico (FIGUEIREDO AEB, et al., 2021).

Em relação as medicações utilizadas, $40,91 \%$ dos idosos utilizam mais de 7 tipos de medicamentos, dado estes foram encontrados no estudo desenvolvido por Souza MC, et al. (2019) com idosos do Centro de Referência da Assistência Social (CRAS) - município de Augustinópolis - TO, onde um número significativo dos idosos apresentaram polifarmácia, ou seja, a maioria fazia uso de mais de três medicamentos, e a grande maioria tinha em suas genealogias pelo menos um caso de doença crônica e/ou degenerativas.

Achados sobre a polifarmácia e a associação entre gênero e comorbidades, corroboram com o estudo de Silva LGC, et al. (2019) que descrevem que a grande maioria dos idosos utilizam vários medicamentos na 
pesquisa realizada em uma Instituição de Longa Permanência Filantrópica sendo exclusivamente masculina do interior do RS e apenas 1,49\% não faziam uso de medicamentos. Os autores enfatizam ainda o predomínio de Doenças Crônica Não Transmissíveis (DCNT) no gênero masculino.

No que se refere a ocorrência de quedas, a pesar da maioria dos idosos serem dependentes de equipamentos para locomoção ou serem acamados, 95,45\%, não obtiveram quedas, nos últimos seis meses, achados que diferem dos achados no estudo de Freitas DCCV, et al, (2018) realizados com grupos de idosos, na Região Centro-Oeste do Brasil no período de 2006 a 2012. Onde mais da metade dos pesquisados sofreram quedas, autores descrevem ainda sobre o crescimento do número de óbitos relacionados as quedas.

Em relação a utilização de dispositivos para locomoção, 36,36\%, dos residentes da ILPI faz uso de cadeira de rodas e 4,55\% são acamados, dados semelhantes aos achados por Geaquini F, et al. (2017) em estudo realizado com idosos residentes de várias ILP de Passo Fundo - RS, os autores ressaltam que houve um predomínio de idosos que faziam uso de dispositivo de marcha com $50,3 \%$ prevalência de locomoção, $41,7 \%$ utilizavam cadeira de rodas, $24 \%$ eram acamados.

Segundo Amorim JSC, et al. (2021) o aumento da longevidade é responsável por trazer mudanças as pessoas idosas, e dentre elas é comum encontrarmos distúrbios relacionado à marcha e a mobilidade é um aspecto essencial para a independência nas atividades de vida diária dos idosos, muitas vezes este comprometimento é solucionado com algum tipo aparato como bengalas, cadeira de rodas, muletas e andadores.

Alves EFB, et al. (2017) discorrem que existem vários fatores que podem levar os idosos a estarem utilizando os dispositivos de marcha, entre eles o com maior predominância é o medo que cair, os idosos acabam utilizando os dispositivos como meio de mobilidade alternativa.

Viana TCT, et al (2018); destaca que atualmente o aumento da população idosa em nossa sociedade é um problema de saúde que poderá ser ainda maior pois essa faixa etária requer atenção diferenciada de dos setores da sociedade, principalmente das equipes envolvidas na assistência à saúde e essa necessidade se deve às diversas alterações que podem ocorrer durante o processo de envelhecimento.

Souza FJM, et al (2020); salienta que com o aumento da idade, ocorrem mudanças na forma e na função que afetam a qualidade de vida do idoso, impossibilitando-o de realizar as atividades diárias. O processo de envelhecimento é considerado dinâmico, gradual e repleto de alterações físicas e mentais, emocionais ou cognitivas, portanto, essas alterações tornam o idoso mais suscetível e vulnerável a determinados problemas de saúde, bem como ao surgimento de patologias que o afetam diretamente e prejudicam a qualidade de vida desse público.

A amostra deste estudo se caracterizou por ser a maioria do sexo masculino, solteiros, com baixa escolaridade e tempo institucionalização maior que 5 anos e não tem contato com familiares. A maioria dos idosos não tem histórico de quedas em seis meses, e com relação aos fatores clínicos a maioria dos idosos apresenta mais de uma doença crônica e/ou degenerativas e utilizam mais de 7 tipos de medicamentos. Os fatores funcionais evidenciaram comprometimento motor, a maioria utiliza um dispositivo de auxílio para marcha. No entanto o estudo não há associação entre as variáveis, grau de independência (escala de Katz), doenças crônicas e idade.

\section{CONCLUSÃO}

O envelhecimento da população é um processo complexo que ocasiona implicações a toda a sociedade e assegurar aos idosos, que essas transformações advenham com saúde e dignidade é desafiador. Nesse contexto, a institucionalização é vista cada vez mais frequente, como um meio de assistência e abrigo a estes indivíduos, e determinar o perfil dessa população que reside nas ILPI's, vai contribuir para o planejamento e a implementação de intervenções que visem melhorar tanto a qualidade de vida e da assistência, bem como a prevenção e/ou minimização de possíveis perdas funcionais e cognitivas. Os resultados deste trabalho contribuem na determinação da tipologia dessa população e indicam a necessidade de discussão, pesquisa e treinamento por parte da equipe multidisciplinar de saúde para uma melhor assistência. 


\section{REFERÊNCIAS}

1. ALARCON MSF, et al. Violência: circunstancias de ocorrência contra idosos. Rev. Bras. Geriatra. Gerontol., Rio de Janeiro, 2019; 22(6): e190182.

2. ALCÂNTARA RKL, et al. Perfil Sociodemográfico e de Saúde de Idosos Institucionalizados. Rev: enferm UFPE on line., Recife, 2019; 13(3): 674-9.

3. ALVES EFB, et al. Fatores associados ao uso de cadeira de rodas por idosos institucionalizados. Rev: Ciência \& Saúde Coletiva, 2017; 22(11): 3607-3613.

4. AMORIM JSC, et al. Prevalência de queda grave e fatores associados em idosos brasileiros: resultados da Pesquisa Nacional de Saúde, 2013; Ciência \& Saúde Coletiva, 2021; 26(1).

5. ANTUNES C, et al. Atividades de vida diária como preditores cognitivo em idosos institucionalizados. Rev. Portuguesa de Enfermagem de Saúde Mental, 2017; (18).

6. BELEZA CMF, SOARES SM. A concepção de envelhecimento com base na teoria de campo de Kurt Lewin e a dinâmica de grupos. Ciênc. Saúde coletiva, Rio de Janeiro, 2019; 24(8): 3141-3146.

7. CATANEO FM et al. Idosos em Instituição de Longa Permanência e Relações Familiares. Rev. Aten. Saúde, São Caetano do Sul, 2019; 17(60): 28-36.

8. CHINA D et al. Envelhecimento Ativo e Fatores Associados. Revista Kairós: Gerontologia, Revista KairósGerontologia, 2021; 24: 141-156.

9. FLORES JR, et al. Nível de dependência de idosos em instituições de longa permanência. Revista Eletrônica Acervo Saúde, 2021; 13(4).

10. FLUETTI MT, et al. Síndrome da fragilidade em idosos institucionalizados. Rev Bras Geriatr Gerontol; 2018.

11. FIGUEIREDO AEB, et al. Doenças crônicas não transmissíveis e suas implicações na vida de idosos dependentes. Rev: Ciência \& Saúde Coletiva, 2021; 26(1): 77-88.

12. FREITAS, DCCV et al. Dinâmica regional do Centro-Oeste brasileiro e a saúde do idoso: análise dos indicadores de mortalidade por quedas. Rev: Ágora. Santa Cruz do Sul, 2018; 20(02): 67-76.

13. GEAQUINI F et al. Prevalência de dificuldade de locomoção em idosos institucionalizados. Rev: Acta Fisiatr. 2017; 24(1): 1-6.

14. HADDAD PCM, CALAMITA Z. Aspectos sociodemográficos, qualidade de vida e saúde do idoso institucionalizado. Rev enferm UFPE on line. 2020; 14: e243416.

15. LEITE AK et al. Capacidade funcional do idoso institucionalizado avaliado pelo KATZ. Rev. Enfermagem atual Edição Jan. Fev. Mar. 2020; $91(29)$.

16. NASCIMENTO RCRM, et al. Polifarmácia: uma realidade na atenção primária do Sistema Único de Saúde. Rev. Saúde Pública 2017.

17. RIBEIRO EG, et al. Auto percepção de saúde e vulnerabilidade clínico-funcional de idosos de Belo Horizonte/Minas Gerais. Rev. Bras. Enferm. Brasília ,2018; 71(2): 860-867.

18. ROSA VPP, et al. Análise dos fatores de risco para queda em idosos institucionalizados. Rev. bras. geriatr. Gerontol, Rio de Janeiro, 2019; 22(1): e180138.

19. ROHDE J, COUTINHO AS. Vínculos e relações familiares de idosos institucionalizados. Revista Brasileira de Ciências do Envelhecimento Humano, 2020; 17(1).

20. SANTANA JDM, et al. Participação Dos Familiares Na Vida Dos Idosos Institucionalizados No Lar São Francisco De Assis Em Simão Dias/ Se. 2018.

21. SANTOS AO, et al. Qualidade de vida de idosos residentes em Instituição de longa permanência: uma revisão sistemática. Rev. Enfermagem Contemporânea; 2017; 2.

22. SCORTEGAGNA HM, et al. Letramento funcional em saúde de idosos hipertensos e diabéticos atendidos na Estratégia Saúde da Família. Escola Anna Nery 2021.

23. SILVA LGC, et al. Avaliação da funcionalidade e mobilidade de idosos comunitários na atenção primária à saúde. Rev. bras. geriatr. Gerontol. Rio de Janeiro, 2019; 22(5): e190086.

24. SOUZA MC, et al. Qualidade De Vida De Idosos: Um Estudo Com A Terceira Idade. Rev: Temas em Saúde; João Pessoa, 2019; 19(6): 2447-2131.

25. SOUZA FJM, et al. Percepção dos idosos institucionalizados acerca da qualidade de vida; Revista Eletrônica Acervo Saúde; 2020; 12(7): 2178-2091.

26. Viana TCT, et al. Qualidade de vida dos idosos cadastrados no programa hiperdia de uma unidade básica de saúde na região norte. Revista Eletrônica Acervo Saúde, 2018; (19): e224. 